\title{
BOUNDED AND ZERO-CONVERGENT SOLUTIONS OF A CLASS OF STIELTJES INTEGRO-DIFFERENTIAL EQUATIONS
}

\author{
SHAOZHU CHEN, QINGGUANG HUANG, AND L. H. ERBE
}

(Communicated by Kenneth R. Meyer)

\begin{abstract}
We consider asymptotic properties of solutions to a class of nonlinear Stieltjes integro-differential equations. Necessary and sufficient conditions are given which guarantee that there exist solutions which do (or do not) have nonzero limits at $\infty$. These extend earlier results of various authors and apply to linear and nonlinear difference equations as well.
\end{abstract}

\section{INTRODUCTION}

Consider the Stieltjes integro-differential equation

$$
G\left(p(t) x^{\prime}(t)\right)=c+\int_{a}^{t} f(x(s)) d \sigma(s), \quad t \in[a, \infty),
$$

where $p, \sigma:[a, \infty) \rightarrow \mathbb{R}$ are right continuous functions that are locally of bounded variation with $p(t)>0,1 / p(t)$ locally integrable, and $\sigma(t)$ nondecreasing. The functions $f, G: \mathbb{R} \rightarrow \mathbb{R}$ are assumed to be continuous with $x f(x)>0, x G(x)>0$ for $x \neq 0, G(x) \rightarrow \pm \infty$ as $x \rightarrow \pm \infty, f$ nondecreasing and $G$ increasing, $c=G\left(p(a) x^{\prime}(a)\right)$, and $x^{\prime}(t)$ denotes the right derivative of $x(t)$. A solution of (1) is understood to be an absolutely continuous function satisfying (1) whenever it exists. The equivalent Stieltjes integral equation to the initial value problem of (1) with $x(a)=c_{0}$ is

$$
x(t)=c_{0}+\int_{a}^{t} \frac{1}{p(s)} G^{-1}\left(c+\int_{a}^{s} f(x(r)) d \sigma(r)\right) d s .
$$

We refer to [9] for a discussion of existence, uniqueness, continuation, and continuous dependence on parameters of solutions to (2).

Our main concern in this paper is to study boundedness and convergence to zero (or to a nonzero limit) of solutions to (1) as $t \rightarrow \infty$. The results we obtain extend earlier results of Marini and Zecca [6], Cheng, Li, and Patula [4], and Liang and the first author $[2,3,5]$. Because of the formulation as a Stieltjes

Received by the editors May 18, 1990.

1980 Mathematics Subject Classification (1985 Revision). Primary 34C11.

The third author's research was supported by NSERC-Canada. 
integro-differential equation, the results obtained apply to linear and nonlinear difference equations as well as second order ordinary differential equations.

In a recent paper [1] Cecchi, Marini, and Villari considered the nonlinear equation

$$
\left(p(t) x^{\prime}(t)\right)^{\prime}=q(t) f(x(t))
$$

and gave conditions under which there exist solutions that are convergent to zero and, at the same time, solutions that have nonzero limit at infinity. This answered an earlier problem posed in [7] and extended the results for the linear case. Corollary 3 improves further the result of [1] by relaxing assumptions on the function $f$. Whereas the authors of [1] used a fixed point theorem for operators defined by Schauder's linearization device, we are able to obtain a more general result by a more or less standard application of the SchauderTychonov Fixed Point Theorem.

We call $x(t)$ a proper solution of (1) if $x(t)$ is a solution of $(1)$ on $[b,+\infty)$ for some $b \geq a$ and $x(t)$ is not eventually identically equal to zero.

Lemma 1. If $x(t)$ is a solution of (1) on $[a, b]$ and $x(a)=x(b)=0$, then $x(t) \equiv 0, a \leq t \leq b$.

Proof. We have

$$
\int_{a}^{b} x(t) d G\left(p(t) x^{\prime}(t)\right)=\left.x\left(G\left(p x^{\prime}\right)\right)\right|_{a} ^{b}-\int_{a}^{b} x^{\prime} G\left(p x^{\prime}\right) d s=-\int_{a}^{b} x^{\prime} G\left(p x^{\prime}\right) d s .
$$

Also,

and so

$$
\int_{a}^{b} x(t) d G\left(p(t) x^{\prime}(t)\right)=\int_{a}^{b} x(s) f(x(s)) d \sigma(s)
$$

$$
\int_{a}^{b} x(s) f(x(s)) d \sigma(s)+\int_{a}^{b} x^{\prime} G\left(p x^{\prime}\right) d s=0,
$$

which implies $x(t) \equiv 0$.

Corollary 1. If $x(t)$ is a solution of (1) that is not eventually equal to zero, then $x^{\prime}(t)$ changes sign at most once and hence, $x(t)$ is eventually monotone.

From the above, it follows that all proper solutions of (1) are nonoscillatory and, as in [1], can be divided into the following two classes:

$$
\begin{aligned}
& A=\left\{x, \text { a proper solution of }(1): \exists t_{x} \geq b_{x}: x(t) x^{\prime}(t)>0 \text { for } t>t_{x}\right\}, \\
& B=\left\{x, \text { a proper solution of }(1): x(t) x^{\prime}(t) \leq 0 \text { for } t>b_{x}\right\} .
\end{aligned}
$$

$\left(\right.$ Here $\left[b_{x},+\infty\right)$ is the interval of existence for the solution $\left.x=x(t)\right)$. Thus, the solutions in class $A$ are either eventually increasing and positive or eventually decreasing and negative, and those in class $B$ are either nonincreasing and positive or nondecreasing and negative. The asymptotic behavior of solutions of equation (3) that belong to class $(A)$ was studied in [6] and [8], whereas the work in [1] was devoted to a study of the asymptotic behavior of solutions 
in class $(B)$. In this work, as indicated earlier, we examine the asymptotic behavior of solutions of $(1)$ in classes $(A)$ and $(B)$, in $\S \S 2$ and 3 , respectively.

\section{BoundedNESS AND CLASS $(A)$}

We introduce the following improper integrals:

$$
\begin{aligned}
& J_{1}=\lim _{t \rightarrow \infty} \int_{a}^{t} \frac{1}{p(s)} G^{-1}\left(\int_{a}^{s} d \sigma(r)\right) d s \\
& J_{2}=\lim _{t \rightarrow \infty} \int_{a}^{t} \frac{1}{p(s)} G^{-1}\left(-\int_{a}^{s} d \sigma(r)\right) d s
\end{aligned}
$$

We also introduce the conditions:

$\left(\mathrm{H}_{1}\right) \quad \int^{ \pm \infty} \frac{d s}{f(s)}= \pm \infty$.

$\left(\mathrm{H}_{2}\right) \quad\left|G^{-1}(u x)\right| \leq M u\left|G^{-1}(x)\right|, M>0, u>1$, and all $x \in \mathbb{R}$.

$\left(\mathrm{H}_{3}\right) \quad\left|G^{-1}(u v)\right| \leq M\left|G^{-1}(u)\right|\left|G^{-1}(v)\right|, M>0$.

We note that if $\left(\mathrm{H}_{1}\right)$ holds, then it follows that all solutions of (1) can be extended to the right to $\infty$. Motivating examples of functions satisfying $\left(\mathrm{H}_{2}\right)$ (resp. $\left.\left(\mathrm{H}_{3}\right)\right)$ are $G(x)=|x|^{\alpha} \operatorname{sgn} x, \alpha \geq 1$ (resp. $\left.\alpha>0\right)$.

Theorem 1. Let $\left(\mathrm{H}_{1}\right)$ and $\left(\mathrm{H}_{2}\right)$ hold. Then all positive (negative) solutions in class $(A)$ are bounded if and only if $J_{1}<+\infty \quad\left(J_{2}>-\infty\right)$.

Proof. Sufficiency. Let $x(t)$ be a positive solution in class $(A)$ and assume $0<x(t) \rightarrow+\infty$ as $t \rightarrow \infty, x^{\prime}(t)>0, t \geq b \geq a$. Then we have

$$
\begin{aligned}
p(t) x^{\prime}(t) & =G^{-1}\left[c_{b}+\int_{b}^{t} f(x(s)) d \sigma(s)\right] \leq G^{-1}\left[c_{b}+f(x(t)) \int_{b}^{t} d \sigma(s)\right] \\
& \leq G^{-1}\left[f(x(t))\left(c_{1}+\int_{b}^{t} d \sigma(s)\right)\right],
\end{aligned}
$$

where $c_{b}=G\left(p(b) x^{\prime}(b)\right), c_{1}=c_{b} / f(x(b))$. Therefore,

$$
\begin{aligned}
\frac{x^{\prime}(t)}{f(x(t))} & \leq \frac{1}{p(t) f(x(t))} G^{-1}\left[\left(c_{1}+\int_{b}^{t} d \sigma\right) f(x(t))\right] \\
& \leq \frac{1+M f(x(t))}{p(t) f(x(t))} G^{-1}\left(c_{1}+\int_{b}^{t} d \sigma\right) .
\end{aligned}
$$

Since $\sigma(t)$ is nondecreasing, we can choose $k>1$ so that $c_{1}+\int_{b}^{t} d \sigma \leq k \int_{b}^{t} d \sigma$ for $t \geq t_{1} \geq b$ and hence from $\left(\mathrm{H}_{2}\right), x^{\prime}(t) / f(x(t)) \leq\left(c_{2} / p(t)\right) G^{-1}\left(\int_{b}^{t} d \sigma\right)$, $t \geq t_{1}$ where $c_{2}=M k(M+1 / f(x(b)))$. Consequently, we have

$$
\int_{x\left(t_{1}\right)}^{x(t)} \frac{d s}{f(s)} \leq c_{2} \int_{t_{1}}^{t} \frac{1}{p(s)} G^{-1}\left(\int_{b}^{s} d \sigma\right) d s<+\infty,
$$

which by $\left(\mathrm{H}_{1}\right)$, implies that $x(t)$ is bounded. This is a contradiction.

Necessity. Let $x(t)$ be a solution of (1) satisfying $x(a)=1, x^{\prime}(a)>0$. Then $x(t)>0, x^{\prime}(t)>0$ for $t \geq a$ and so $\lim _{t \rightarrow \infty} x(t) \equiv L>0$ and 


$$
\begin{aligned}
f(x(t)) \geq f(1) & >0 . \text { Let } \alpha=\min \{1, f(1) / M\} . \text { Then } \\
x^{\prime}(t) & \geq \frac{1}{p(t)} G^{-1}\left(\int_{a}^{t} f(x(s)) d \sigma\right) \geq \frac{1}{p(t)} G^{-1}\left(f(1) \int_{a}^{t} d \sigma\right) \\
& \geq \frac{\alpha}{p(t)} G^{-1}\left(\int_{a}^{t} d \sigma\right) .
\end{aligned}
$$

A quadrature now gives $\int_{a}^{\infty}(1 / p(s)) G^{-1}\left(\int_{a}^{s} d \sigma\right) d s=J_{1}<+\infty$. Similar arguments hold for negative solutions in class $(A)$.

Remark. We observe, therefore, that if $\left(\mathrm{H}_{1}\right),\left(\mathrm{H}_{2}\right)$ hold, then all solutions of (1) are bounded if and only if the two integrals in (4) converge.

Corollary 2. Let $\left(\mathrm{H}_{1}\right),\left(\mathrm{H}_{2}\right)$ hold. If (1) has a positive (negative) solution in class $(A)$ that is bounded, then all positive (negative) solutions in class $(A)$ are bounded. Conversely, if (1) has an unbounded positive (negative) solution in class $(A)$, then all positive (negative) solutions in class $(A)$ are unbounded.

We note that in the proof of the necessity in Theorem 1 , the hypothesis $\left(\mathrm{H}_{1}\right)$ was not used. Without $\left(\mathrm{H}_{1}\right)$ we still have

Theorem 2. Let $\left(\mathrm{H}_{2}\right)$ or $\left(\mathrm{H}_{3}\right)$ hold. Equation (1) has a bounded positive (negative) solution in class $(A)$ if and only if $J_{1}<+\infty \quad\left(J_{2}>-\infty\right)$.

Proof. We only prove the sufficiency for the case $J_{1}<+\infty$. It suffices to show that the equation

$$
x(t)=1-\int_{t}^{\infty} \frac{1}{p(s)} G^{-1}\left(\int_{b}^{s} f(x(r)) d \sigma(r)\right) d s, \quad t \geq b
$$

has a solution, where $b \geq a$ is chosen so that

$$
\int_{b}^{\infty} \frac{1}{p(s)} G^{-1}\left(\int_{b}^{s} d \sigma\right) d s<1 / \alpha
$$

with

$$
\alpha= \begin{cases}1+M f(1) & \text { if }\left(\mathrm{H}_{2}\right) \text { holds } \\ M G^{-1}(f(1)) & \text { if }\left(\mathrm{H}_{3}\right) \text { holds } .\end{cases}
$$

We define a set

$$
E=\{x \in C[b, \infty): 0 \leq x(t) \leq 1, t \geq b\}
$$

and an operator $T: E \rightarrow C[b, \infty)$ by

$$
(T x)(t):=1-\int_{t}^{\infty} \frac{1}{p(s)} G^{-1}\left(\int_{b}^{s} f(x(r)) d \sigma(r)\right) d s .
$$

It is not difficult to show that $T$ maps $E$ into $E$ and that $T$ is continuous and compact under the topology generated by uniform convergence on compact subsets of $[b, \infty)$. By the Schauder-Tychonov Fixed Point Theorem, $T$ has a fixed point $x$ in $E$, which is a solution of (5). 


\section{ZERO CONVERGENT SOLUTIONS AND CLASS $(B)$}

In this section we always suppose that $f(x)$ satisfies a local Lipschitz condition for $x \neq 0$. Thus, any IVP of (1) with nonzero initial value of $x(t)$ has a unique local solution, and this solution is continuously dependent on initial data on its existence interval where it does not vanish. We write $B=B_{0} \cup B_{L}$, where $B_{0}$ denotes the set of solutions of (1) that belong to $B$ and satisfy $x(+\infty)=0$ and $B_{L}=B \backslash B_{0}$ is the set of solutions in $B$ with $x(+\infty) \neq 0$. We are interested in establishing conditions under which $B, B_{0}, B_{L}$ are (or are not) empty. These extend and improve the results of $[1,5]$. To this end, we introduce the condition

$$
\left(\mathrm{H}_{4}\right) \quad \liminf _{x \rightarrow 0} G^{-1}(f(x)) / x<\infty .
$$

Theorem 3. For each $b \geq a$ and for each $x_{0}>0 \quad\left(x_{0}<0\right)$ equation (1) has a nonincreasing (nondecreasing) and nonnegative (nonpositive) solution $x(t)$ on $[b, \infty)$ satisfying $x(b)=x_{0}$. Further, if $\left(\mathrm{H}_{3}\right),\left(\mathrm{H}_{4}\right)$ hold, then the above solution belongs to class $B$.

Proof. We use a shooting-type method and consider the case $x_{0}>0$ only. We denote by $x(t):=x(t, c)$ the solution of equation (1) with $x(b)=x_{0}$ and $p(b) x^{\prime}(b)=c$. Then $x(t, c)$ satisfies

$$
x(t)=x_{0}+\int_{b}^{t} \frac{d s}{p(s)} G^{-1}\left(G(c)+\int_{b}^{s} f(x(r)) d \sigma(r)\right) .
$$

Let $U, L$ be defined by

$$
\begin{aligned}
& U=\left\{c \in \mathbb{R}: \text { there exists } t \geq b \text { such that } x^{\prime}(t, c)>0\right\}, \\
& L=\{c \in \mathbb{R}: \text { there exists } t \geq b \text { such that } x(t, c)<0\} .
\end{aligned}
$$

From the corollary to Lemma 1 , it follows that $U \cap L=\varnothing$. Clearly, we have $U \neq \varnothing$, and if $c_{0} \in U$, it follows that $\left[c_{0}, \infty\right) \subset U$. We claim that $U$ is open. If $c_{0} \in U$ then there exists $t_{0}>b$ such that $x^{\prime}\left(t_{0}, c_{0}\right)>0$; so from (1) we have

$$
\begin{aligned}
& G\left(p\left(t_{0}\right) x^{\prime}\left(t_{0}, c_{0}\right)\right)-G\left(p\left(t_{0}\right) x^{\prime}\left(t_{0}, c\right)\right) \\
& \quad=G\left(c_{0}\right)-G(c)+\int_{b}^{t_{0}}\left[f\left(x\left(s, c_{0}\right)\right)-f(x(s, c))\right] d \sigma(s) .
\end{aligned}
$$

Continuous dependence on initial data implies that for all $c$ sufficiently close to $c_{0}$, we have $x(t, c)>0$ for $b \leq t \leq t_{0}$ and $f\left(x\left(s, c_{0}\right)\right)-f(x(s, c)) \rightarrow 0$ as $c \rightarrow c_{0}$, uniformly for $s \in\left[b, t_{0}\right]$. Hence, $x^{\prime}\left(t_{0}, c\right)>0$ for all $c$ close to $c_{0}$, so that $U$ is open.

We next show that $L \neq \varnothing$. Let $A=\int_{b}^{b+1} d s / p(s), B=\int_{b}^{b+1} d \sigma(s)$, and choose $c$ such that $G(c)<G\left(-x_{0} / A\right)-f\left(x_{0}\right) B$. Then $x^{\prime}(t, c)<0$ for $b \leq$ $t \leq b+1$ since otherwise there exist $s \in(b, b+1]$ for which $x^{\prime}(s, c)=0$ and 
$x^{\prime}(t, c)<0, t \in[b, s)$. But then we have

$$
\begin{aligned}
0=G\left(p(s) x^{\prime}(s, c)\right) & =G(c)+\int_{b}^{s} f(x(r, c)) d \sigma(r) \\
& <G\left(-\frac{x_{0}}{A}\right)-f\left(x_{0}\right) B+f\left(x_{0}\right) \int_{b}^{s} d \sigma \\
& \leq G\left(-\frac{x_{0}}{A}\right)<0,
\end{aligned}
$$

a contradiction. Thus, $x(t, c)$ is decreasing on $[b, b+1]$. From (2) we have

$$
\begin{aligned}
x(b+1, c) & <x_{0}+\int_{b}^{b+1} \frac{d s}{p(s)} G^{-1}\left(G\left(-\frac{x_{0}}{A}\right)-f\left(x_{0}\right) B+f\left(x_{0}\right) \int_{b}^{s} d \sigma\right) \\
& \leq x_{0}+A\left(-\frac{x_{0}}{A}\right)=0
\end{aligned}
$$

and hence $c \in L$. Also, $L$ is open. Thus, $V:=\mathbb{R}-(U \cup L) \neq \varnothing$ and for all $c \in V, x(t, c)$ is a nonincreasing nonnegative solution on $[b, \infty)$.

Next assume that $\left(\mathrm{H}_{3}\right),\left(\mathrm{H}_{4}\right)$ hold. We show that $x(t)=x(t, c)>0$ for $c \in V$. If not, then there exists $t_{0}>b$ with $x\left(t_{0}\right)=0$ and $x(t) \equiv 0$ for $t \geq t_{0}$, $x^{\prime}\left(t_{0}\right)=0$ (right derivative). From (1) we have

$$
G\left(p(b) x^{\prime}(b)\right)=-\int_{b}^{t_{0}} f(x(s)) d \sigma(s)
$$

so for $t \in\left[b, t_{0}\right]$, we have

$$
G\left(p(t) x^{\prime}(t)\right)=-\int_{t}^{t_{0}} f(x(s)) d \sigma(s)
$$

and by $\left(\mathrm{H}_{3}\right)$,

$$
\begin{aligned}
x(t) & =-\int_{t}^{t_{0}} \frac{1}{p(s)} G^{-1}\left(-\int_{s}^{t_{0}} f(x(r)) d \sigma(r)\right) d s \\
& \leq-\int_{t}^{t_{0}} \frac{d s}{p(s)} G^{-1}\left(-f(x(s)) \int_{s}^{t_{0}} d \sigma(r)\right) \\
& \leq-M \int_{t}^{t_{0}} \frac{d s}{p(s)} G^{-1}(f(x(s))) G^{-1}\left(-\int_{s}^{t_{0}} d \sigma\right) \\
& \leq M G^{-1}\left(f(x(t)) \int_{t}^{t_{0}} \frac{d s}{p(s)}\left|G^{-1}\left(-\int_{s}^{t_{0}} d \sigma\right)\right| .\right.
\end{aligned}
$$

Now by $\left(\mathrm{H}_{4}\right)$, there exists an increasing sequence $t_{n} \rightarrow t_{0}, n \rightarrow \infty$, and $N>0$ such that

$$
x\left(t_{n}\right) \leq M N x\left(t_{n}\right) \int_{t_{n}}^{t_{0}} \frac{d s}{p(s)}\left|G^{-1}\left(-\int_{s}^{t_{0}} d \sigma\right)\right| .
$$

But this is impossible since $1 / p$ is locally integrable. Thus, $x(t)>0, t \geq b$ and therefore, belongs to class $B$. This completes the proof of Theorem 3 . 
If $\int^{\infty} d \sigma(t)<+\infty$, then we denote

$$
\begin{aligned}
& J_{3}=\int_{a}^{\infty} \frac{1}{p(s)} G^{-1}\left(\int_{s}^{\infty} d \sigma(r)\right) d s, \\
& J_{4}=\int_{a}^{\infty} \frac{1}{p(s)} G^{-1}\left(-\int_{s}^{\infty} d \sigma(r)\right) d s .
\end{aligned}
$$

In the next result, we characterize the case $B_{L} \neq \varnothing$ under $\left(\mathrm{H}_{3}\right)$, in analogy with Theorem 4 in [5] where the following equation was considered:

$$
x^{\prime \prime}(t)=X\left(t, x(t), x^{\prime}(t)\right) .
$$

Theorem 4. Let $\left(\mathrm{H}_{3}\right)$ hold. Then there exists a positive (negative) solution in $B_{L}$ if and only if $\int^{\infty} d \sigma(t)<+\infty$ and $J_{4}>-\infty\left(J_{3}<+\infty\right)$.

Proof. Necessity. Let $x=x(t) \in B$ and assume $x_{1}:=\lim _{t \rightarrow \infty} x(t)>0$. Then $x(t) \geq x_{1}$ for $t>b_{x}$. Since $p(t) x^{\prime}(t) \leq 0$ and $p(t) x^{\prime}(t)$ is nondecreasing, it follows that $\xi:=\lim _{t \rightarrow \infty} G\left(p(t) x^{\prime}(t)\right) \leq 0$ exists. Then from (1), we have

$$
G\left(p(t) x^{\prime}(t)\right)=\xi-\int_{t}^{\infty} f(x(s)) d \sigma(s)
$$

and $\int^{\infty} d \sigma(t)<+\infty$. Integrating (7) gives

$$
x(t)=x_{1}-\int_{t}^{\infty} \frac{d s}{p(s)} G^{-1}\left(\xi-\int_{s}^{\infty} f(x(r)) d \sigma(r)\right) .
$$

Thus from (8) and $\left(\mathrm{H}_{3}\right)$, we have

$$
\begin{aligned}
x(t) & \geq x_{1}-\int_{1}^{\infty} \frac{d s}{p(s)} G^{-1}\left(-f\left(x_{1}\right) \int_{s}^{\infty} d \sigma\right) \\
& \geq x_{1}-\frac{1}{M G^{-1}\left(1 / f\left(x_{1}\right)\right)} \int_{t}^{\infty} \frac{d s}{p(s)} G^{-1}\left(-\int_{s}^{\infty} d \sigma\right)
\end{aligned}
$$

and hence $J_{4}>-\infty$. The proof for the case $x_{1}<0$ is similar.

Sufficiency. Assume that $J_{4}>-\infty$. We consider the integral equation

$$
x(t)=1-\int_{t}^{\infty} \frac{1}{p(s)} G^{-1}\left(-\int_{s}^{\infty} f(x(r)) d \sigma(r)\right) d s .
$$

A similar argument used in the proof of Theorem 2 shows that (9) has a solution $x$ on $[b, \infty)$ for a suitably large $b$. Clearly, $x(t)$ is a positive solution in $B_{L}$. The case $J_{3}<+\infty$ can be handled in the same way.

Theorem 5. Let $\left(\mathrm{H}_{3}\right),\left(\mathrm{H}_{4}\right)$ hold. If $\int^{\infty} d s / p(s)<+\infty$, then there exist both positive and negative solutions in $B_{0}$.

Proof. Theorem 3 shows that equation (1) has a solution $x(t)>0$ on $[a, \infty)$ with $x(a)=1$. Then $x(t)$ satisfies (7). Assume that $x_{1}:=\lim _{t \rightarrow \infty} x(t)>0$. By Theorem 4 we have $J_{4}>-\infty$. Let

$$
E=\{y \in C[a, \infty): 0 \leq y(t) \leq x(t), t \geq a\},
$$


and let $T: E \rightarrow C[a, \infty)$ be the operator defined by

$$
(T y)(t):=-\int_{t}^{\infty} \frac{d s}{p(s)} G^{-1}\left(\xi-\delta-\int_{s}^{\infty} f(y(r)) d \sigma(r)\right),
$$

where $\xi \leq 0$ is the constant in (7) and $\delta>0$ is a constant to be determined later. Clearly we have $(T y)(t)>0$ for all $t \geq a, y \in E$. From (7),

$$
0 \leq \int_{t}^{\infty} f(x(s)) d \sigma(s) \leq \xi-G\left(p(a) x^{\prime}(a)\right):=\xi-c .
$$

Now by the uniform continuity of $G^{-1}(y)$ on any compact interval of $\mathbb{R}$, we can choose $\delta>0$ so small that

$$
\max _{c \leq s \leq \xi}\left\{G^{-1}(s)-G^{-1}(s-\delta)\right\} \leq x_{1}\left[\int_{a}^{\infty} \frac{d s}{p(s)}\right]^{-1} .
$$

Then

$$
\begin{aligned}
x(t)-(T y)(t)= & x_{1}-\int_{t}^{\infty} \frac{d s}{p(s)}\left[G^{-1}\left(\xi-\int_{s}^{\infty} f(x) d \sigma\right)\right. \\
& \left.-G^{-1}\left(\xi-\delta-\int_{s}^{\infty} f(y) d \sigma\right)\right] \\
= & x_{1}-\int_{t}^{\infty} \frac{d s}{p(s)}\left[G^{-1}\left(\xi-\int_{s}^{\infty} f(x) d \sigma\right)\right. \\
& \left.-G^{-1}\left(\xi-\int_{s}^{\infty} f(x) d \sigma-\delta\right)\right] \\
& -\int_{t}^{\infty} \frac{d s}{p(s)}\left[G^{-1}\left(\xi-\int_{s}^{\infty} f(x) d \sigma-\delta\right)\right. \\
\geq & x_{1}-\int_{t}^{\infty} \frac{d s}{p(s)} \max _{c \leq s \leq \xi}\left\{G^{-1}(s)-\int_{s}^{-1}(s-\delta)\right\} \geq 0 .
\end{aligned}
$$

Hence, $T$ maps $E$ into $E$. It is easy to show that $T$ is continuous and compact under the topology mentioned in the proof of Theorem 2 . Thus $T$ has a fixed point $y \in E$ for which

$$
y(t)=-\int_{t}^{\infty} \frac{1}{p(s)} G^{-1}\left(\xi-\delta-\int_{s}^{\infty} f(y(r)) d \sigma(r)\right), \quad t \geq a,
$$

and $y(t)$ is a decreasing proper solution approaching zero. The proof of the existence of a negative solution in $B_{0}$ is similar.

We observe that Theorem 6 in [1] (see also [7]) shows that for equation (3) the class $B$ is not empty if a condition stronger than $\left(\mathrm{H}_{4}\right)$ holds:

$\left(\mathrm{H}_{5}\right) \quad$ There exists $h>0$ such that $\sup _{|r| \leq h} f(r) / r<+\infty$.

Theorem 3 improves this by requiring only that $\left(\mathrm{H}_{4}\right)$ holds. Theorem 5 shows, more precisely, that $B_{0} \neq \varnothing$ if $\int^{\infty} d s / p(s)<+\infty$ (in addition to $\left.\left(\mathrm{H}_{4}\right)\right)$. Moreover, if $B \neq \varnothing$ (e.g. if $\left(\mathrm{H}_{4}\right)$ holds), from Theorem 4 we know 
that under the hypothesis $\left(\mathrm{H}_{3}\right), B=B_{0}$ if and only if either $\int^{\infty} d \sigma(t)=+\infty$ or $J_{3}=+\infty$ and $J_{4}=-\infty$.

The next result is a complementary result to Theorem 4 and gives a necessary and sufficient condition in order that $B=B_{L}$ under $\left(\mathrm{H}_{3}\right),\left(\mathrm{H}_{4}\right)$, in analogy with Theorem 5 in [5] (where equation (6) was studied).

Theorem 6. Let $\left(\mathrm{H}_{3}\right),\left(\mathrm{H}_{4}\right)$ hold. Then all positive (negative) solutions in $B$ are in $B_{L}$ if and only if $\int^{\infty} d t / p(t)=+\infty, \int^{\infty} d \sigma(t)<\infty$ and $J_{4}>-\infty$ $\left(J_{3}<+\infty\right)$.

Proof. By Theorem 3 we have $B \neq \varnothing$. Thus, the necessity follows from Theorems 4 and 5.

Let $x(t)$ be any positive solution in $B$ and assume further that $J_{4}>-\infty$. Then $x(t)$ satisfies (7) with $\xi=0$ since $\int^{\infty} d t / p(t)=+\infty$. If $x \in B_{0}$, then we have

$$
\begin{aligned}
x(t) & =-\int_{t}^{\infty} \frac{d s}{p(s)} G^{-1}\left(-\int_{s}^{\infty} f(x(r)) d \sigma(r)\right) \\
& \leq M G^{-1}(f(x(t))) \int_{t}^{\infty} \frac{d s}{p(s)}\left[-G^{-1}\left(-\int_{s}^{\infty} d \sigma\right)\right] .
\end{aligned}
$$

By $\left(\mathrm{H}_{4}\right)$ there exists a sequence $t_{n} \rightarrow+\infty$ such that $G^{-1}\left(f\left(x\left(t_{n}\right)\right)\right) \leq N x\left(t_{n}\right)$ for some $N>0$ and so

$$
x\left(t_{n}\right) \leq x\left(t_{n}\right) M N \int_{t_{n}}^{\infty} \frac{d s}{p(s)}\left[-G^{-1}\left(-\int_{s}^{\infty} d \sigma\right)\right],
$$

which is impossible for large $n$. Thus we have $x \in B_{L}$. For negative solutions in $B$, the proof is similar.

In our final result, we characterize the case $B_{L} \neq \varnothing \neq B_{0}$ under $\left(\mathrm{H}_{3}\right),\left(\mathrm{H}_{4}\right)$. Corollary 3. Let $\left(\mathrm{H}_{3}\right),\left(\mathrm{H}_{4}\right)$ hold. Then both $B_{L}$ and $B_{0}$ contain positive and negative solutions if and only if $\int^{\infty} d t / p(t)<+\infty, \int^{\infty} d \sigma(t)<+\infty$.

Proof. Since $\int^{\infty} d t / p(t)<+\infty$ and $\int^{\infty} d \sigma(t)<+\infty$ imply that $J_{3}<+\infty$ and $J_{4}>-\infty$, Corollary 3 now follows from Theorems 4,5 , and 6 .

For equation (3), Theorem 8 in [1] shows that if $\left(\mathrm{H}_{5}\right)$ holds and

$$
\begin{aligned}
& \int_{a}^{+\infty} \frac{1}{p(s)} \int_{a}^{s} q(r) d r d s<+\infty \\
& \int_{a}^{+\infty} q(s) \int_{a}^{s} \frac{1}{p(r)} d r d s<+\infty
\end{aligned}
$$

then $B_{L} \neq \varnothing \neq B_{0}$. Obviously, (10) and (11) hold if and only if $\int^{\infty} d s / p(s)<$ $+\infty$ and $\int^{\infty} q(s) d s<+\infty$. Therefore, even for equation (3), Corollary 3 improves this by requiring only that $\left(\mathrm{H}_{4}\right)$ holds and proves the necessity of (10), (11). 


\section{REFERENCES}

1. M. Cecchi, M. Marini, and G. Villari, On the monotonicity property for a certain class of second order differential equations, J. Differential Equations 82 (1989), 15-27.

2. S. Chen, Terminal value problems for $x^{\prime \prime}=f\left(t, x, x^{\prime}\right)$, Ann. Differential Equations 5 (1989), 389-395.

3. S. Chen, Existence and uniqueness of solutions of limit boundary value problems for second order differential systems, Acta Math. Appl. Sinica 10 (1987), 324-332.

4. S. S. Cheng, H. J. Li and W. T. Patula, Bounded and zero convergent solutions of second order differential equations, preprint.

5. Z. Liang and S. Chen, Asymptotic behavior of solutions to second order nonlinear differential equations, Chinese Ann. Math. Ser. B 6 (1985), 481-490.

6. M. Marini and P. Zecca, On the asymptotic behavior of the solutions of a class of second order linear differential equations, J. Differential Equations 28 (1978), 1-17.

7. M. Marini, On nonoscillatory solutions of a second order nonlinear differential equation, Boll. Un. Mat. Ital. C (6) (1984), 189-202.

8. _ Monotone solutions of a class of second order nonlinear differential equations, Nonlinear Anal. 8 (1984), 261-271.

9. B. You and Q. Huang, Fundamental theory of a class of functional equations and its applications in the study of Kreser's theorem, Ann. Differential Equations 5 (1989), 107-128.

(Shaozhu Chen and Qingguang Huang) Department of Mathematics, Shandong UniverSity, Jinan, Shandong 250100 People's Republic of China

(L. H. Erbe) Department of Mathematics, University of Alberta, Edmonton, Alberta T6G 2G1 Canada 